\title{
Use of a fixed, body weight-unadjusted loading dose of unfractionated heparin for extracorporeal cardiopulmonary resuscitation
}

\author{
Yoshiaki Iwashita*, Mashiro Yukimitsu, Masaki Matsuduki, Akitaka Yamamoto, Ken Ishikura and Hiroshi Imai
}

\begin{abstract}
Background: Extracorporeal cardiopulmonary resuscitation (ECPR) is being used increasingly in the emergency and critical care field in Japan. A major complication of ECPR is bleeding; however, the optimal initial heparin dose and activated coagulation time (ACT) remain unknown. The aim of this study was to assess the appropriateness of our initial anticoagulation protocol.

Methods: We retrospectively evaluated the initial heparin dose, ACT value, and incidence of bleeding and thrombotic complications in post-cardiopulmonary arrest patients who received a fixed, body weight-unadjusted loading dose of unfractionated heparin (3000 U) prior to veno-arterial extracorporeal membrane oxygenator (ECMO) between February 2011 and November 2013 at Mie University Hospital, Japan.

Results: ACT was evaluated within $3 \mathrm{~h}$ of initiation of 32 consecutive ECPR patients. The mean heparin dose per body weight was $53.6 \mathrm{U} / \mathrm{kg}$ and the mean ACT was $231.3 \mathrm{~s}$. In 17 patients, ACT exceeded $200 \mathrm{~s}$. Three patients experienced fatal bleeding in the chest wall within $24 \mathrm{~h}$ of receiving ECMO. The mean heparin dose per kilogram body weight, mean initial ACT, and mean duration of cardiopulmonary resuscitation (CPR) did not statistically differ between the patients who experienced fatal bleeding and those who did not.

Conclusions: Fixed-dose heparin of 3000-U bolus resulted in a mean heparin dose per kilogram body weight of $53.6 \mathrm{U} / \mathrm{kg}$ and an ACT of $231.3 \mathrm{~s}$ and experienced 3 out of 32 fatal bleedings. Further researches are warranted to optimize anticoagulation protocol for ECPR patients.
\end{abstract}

Keywords: Heparin, Extracorporeal cardiopulmonary resuscitation, Activated coagulation time

\section{Background}

Veno-arterial extracorporeal membrane oxygenation (ECMO) is being used increasingly for extracorporeal cardiopulmonary resuscitation (ECPR) in Japan because it provides higher survival rates and better neurological outcomes $[1,2]$. Despite the development of this new technique, bleeding remains a major complication associated with ECPR. In patients receiving ECMO, anticoagulants such as unfractionated heparin are frequently used. In patients starting respiratory ECMO, the Extracorporeal Life Support Organization (ELSO) recommends 50-100 U/kg of unfractionated heparin given as a bolus injection and a target activated coagulation time (ACT) of 1.5 times the

\footnotetext{
*Correspondence: iwaci1ta@clin.medic.mie-u.ac.jp

Emergency and Critical Care Center, Mie University Hospital, Edobashi 2-174, Tsu, Mie, Japan
}

normal value [3]. Ideally, the heparin dose should be carefully adjusted by referencing both the patient's body weight and normal ACT value; however, in the emergency setting, it is extremely difficult to obtain these values. Therefore, for practical reasons, at our hospital, we decided to use a fixed, body weight-unadjusted loading dose of $3000 \mathrm{U}$ of unfractionated heparin in all patients undergoing ECPR.

To our knowledge, no studies examining the optimal initial heparin loading dose and ACT for veno-arterial ECMO have been conducted. Therefore, we retrospectively assessed the initial heparin loading protocol for veno-arterial ECMO used at our hospital. To achieve this objective, we firstly analysed the relationship between heparin dose and ACT value in our hospital by comparing with ELSO's recommendation. Secondly, we 
analysed the major bleeding complication in the study period.

\section{Methods}

We retrospectively investigated patients who had experienced cardiopulmonary arrest and in whom venoarterial ECMO was initiated at Mie University Hospital, Japan, between February 2011 and November 2013. The study protocol was approved by the institutional review board of Mie University Hospital.

A CAPIOX (Terumo, Tokyo, Japan) ECMO system with a heparin-coated pump and cannulae was used in all of the study patients. A 12- to 18-Fr arterial cannula and a $20-$ Fr venous cannula were used in adult patients. Twelve- or fourteen-Fr cannulae were used in paediatric patients $(<15$ years old $)$.

Upon cardiopulmonary arrest, conventional cardiopulmonary resuscitation (CPR) was initiated in all of the study patients in accordance with the American Heart Association Guidelines for CPR and ECC (2010) without the use of any adjunct devices in either the pre-hospital or hospital setting [4, 5]. Indications for introducing ECMO were age less than 75 years and cardiac arrest time less than $60 \mathrm{~min}$, and ECMO was initiated when requested by one of our Board of Emergency Medicinecertified physicians. Percutaneous cannulation was performed in the emergency room by using the Seldinger technique.

All the patients underwent hypothermia therapy (target body temp., $34{ }^{\circ} \mathrm{C}$ ) for at least $24 \mathrm{~h}$ and were gradually rewarmed. Normal blood pressure was maintained by adjusting the flow of blood through the ECMO pump and by minimizing the use of inotrope and vasopressor agents. Intra-aortic balloon pumps were placed in patients with low pulse pressures. Continuous renal replacement therapy was initiated for all renal indications. Ventilator settings used were "lung rest"; positive end-expiratory pressure, $5-10 \mathrm{~cm} \mathrm{H}_{2} \mathrm{O}$; low respiratory rate $(<12 / \mathrm{min})$; and $\mathrm{FiO}_{2}<60 \%$.

A fixed, body weight-unadjusted loading dose of $3000 \mathrm{U}$ of unfractionated heparin was administered to all the study patients, and unfractionated heparin was used for anticoagulation therapy during ECMO. ACT was evaluated by using a Hemochron microcoagulation system (Technidyne, New Albany, USA). ACT and body weight were determined upon admission to the intensive care unit. In three patients, body weight could not be determined due to the patient's clinical status or death, so body weight was estimated from their age according to the Japanese Ministry of Health, Labour, and Welfare guidelines.

We recorded patient age, sex, initial heparin dose, and initial ACT value; incidence of bleeding or thrombotic complications occurring within $24 \mathrm{~h}$ of ECMO initiation; and survival and neurological outcomes after hospital discharge. Fatal bleeding was defined as any bleeding that required surgical or trans-arterial embolization to maintain hemodynamic stability; minor bleeding was defined as any bleeding that did not require surgical intervention. Thrombotic complications were assessed by visually checking for circuit clotting. Neurological outcomes were assessed by using the Cerebral Performance Category scale.

Continuous variables are presented as mean (range), and categorical variables are presented in the text as percentages. Continuous variables were compared between groups by using the Student's $t$ test. Categorical variables were compared by using the chi-square test. For all analyses, significance was defined as $P<0.05$. All analyses were performed with statistical software (Statistical Package for the Social Sciences [SPSS] version 18; SPSS Japan Inc., Tokyo, Japan).

\section{Results}

The anticoagulation protocol currently used in our hospital is shown in Table 1. The ECMO circuit is primed by using a fixed, body weight-unadjusted loading dose of $3000 \mathrm{U}$ of heparin-infused normal saline. If cannulation takes longer than usual, an additional dose of heparin is administered. Two patients in the present study received a total of $7500 \mathrm{U}$ of heparin, and one patient received a total of $5500 \mathrm{U}$ of heparin. All other patients received $3000 \mathrm{U}$ of heparin.

ECMO was initiated in 45 patients during the study period (Fig. 1). In one patient, ECMO was initiated twice during the same hospitalization. In all other patients, ECMO was initiated once at each hospitalization. ACT was not measured within $3 \mathrm{~h}$ of admission to the intensive care unit in 13 patients so they were excluded from the study. Table 2 shows the patient demographics. The mean age was 60.4 years. Twenty-seven patients $(84.3 \%)$ were male. Nine patients $(37.5 \%)$ experienced cardiac arrest during hospitalization. The mean duration of conventional CPR prior to the initiation of ECMO was $48.6 \mathrm{~min}$. The mean initial heparin dose was $53.6 \mathrm{U} / \mathrm{kg}$ body weight, and the mean initial ACT was $231.3 \mathrm{~s}$.

Nine out of the thirty-two patients $(28.1 \%)$ survived to discharge, six of whom had a good neurological outcome (Cerebral Performance Category 1 or 2). The

Table 1 Comparison of anticoagulation protocols

\begin{tabular}{lll}
\hline & Our protocol & ELSO guideline \\
\hline Initial dose & $3000 \mathrm{U}^{\mathrm{a}}$ & $50-100 \mathrm{U} / \mathrm{kg}$ \\
Additional dose & $2000-5000 \mathrm{U}$ & $\mathrm{NA}$ \\
Target ACT & $180-200 \mathrm{~s}$ & 1.5 times the normal value \\
\hline
\end{tabular}

ACT activated coagulation time, ELSO Extracorporeal Life Support Organization, NA not applicable

${ }^{\mathrm{a}}$ Fixed and body weight unadjusted 


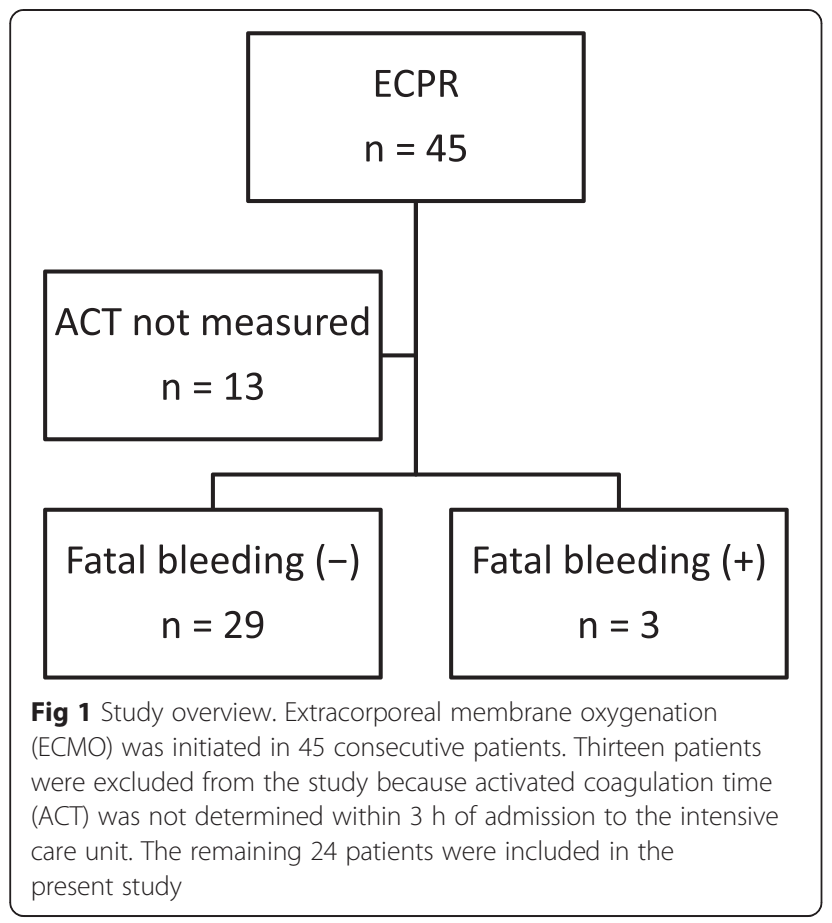

causes of death in the patients that died included cerebral hypoxia, low cardiac output syndrome, and multiple organ failure. Three patients experienced fatal bleeding during ECMO (Table 3), two of whom died as a consequence. The remaining patient underwent interventional radiology to stop the haemorrhage but died due to low cardiac output syndrome. Figure 2 shows computed tomography scans of the three patients who experienced fatal bleeding; thoracic, intra-thoracic, and mediastinal bleeding can be seen. There were no significant differences in age $(P=0.33)$, sex $(P=0.84)$, mean initial heparin dose $(P=0.42)$, mean initial ACT $(P=0.89)$, or mean duration of conventional CPR $(P=0.18)$ between the patients who experienced fatal bleeding and those who did not (Table 3). No significant thrombotic complications were observed.

Table 2 Patient demographics

\begin{tabular}{ll}
\hline Number of patients & 32 \\
Mean age & $60.4(12-87)$ \\
Sex (male to female ratio) & $27: 5$ \\
Mean initial heparin dose (U/kg) & $53.6(31.6-115.2)$ \\
Mean initial ACT (s) & $231.3(103-359)$ \\
Mean duration of conventional CPR (min) & $48.6(4-106)$ \\
Survival & 9 \\
CPC 1 or 2 & 6 \\
\hline
\end{tabular}

\section{Discussion}

It is difficult to measure body weight prior to initiating ECMO in the emergency setting, so at our hospital, we decided to use a fixed, body weight-unadjusted loading dose of heparin as anticoagulation therapy. Therefore, all the patients received $3000 \mathrm{U}$ of heparin; three patients received an additional dose of heparin. As a result, the initial mean heparin dose per kilogram body weight was $53.6 \mathrm{U} / \mathrm{kg}$ and ACT of $231.3 \mathrm{~s}$, and experienced 3 out of 32 fatal bleedings. Although the ELSO guideline recommends a heparin bolus injection of $50-100 \mathrm{U} / \mathrm{kg}$, there is no definitive evidence showing that this dose is sufficient to minimize mortality or morbidity [3]. Indeed, a recently published multicentre prospective trial did not use a fixed anticoagulation protocol for starting ECPR [1]. Furthermore, the ELSO guideline was originally intended for respiratory ECMO, so the appropriate dose of initial heparin for ECPR patients remains unknown.

According to the ELSO database, the currently recommended ACT value, 150-180 s, resulted in a rate of bleeding complications of 30-35\%; however, these data were obtained in the respiratory ECMO patients only and more than $50 \%$ of these patients were venovenously cannulated [6]. Patients who undertake percutaneous coronary intervention (PCI) are recommended to receive $70-100 \mathrm{U} / \mathrm{kg}$ of unfractionated heparin and resultant ACT of $>250 \mathrm{~s}$ before PCI [7]. One of the reasons that ECMO patients require a lower dose of heparin with a shorter ACT value may be due to patient' disease state. Indeed, in PCI patients, the severity of disease state is correlated with the incidence of post PCI bleeding [8]. Patients who need ECMO are usually critically ill with deteriorated coagulation status. Therefore, a study specifically examining anticoagulation strategy for ECPR patients is needed.

Postcardiotomy patients receiving veno-arterial ECMO frequently experience bleeding complications. Rastan et al. analysed 517 consecutive postcardiotomy cardiogenicshock patients who required extracorporeal circulation and found that 300/517 (58.0\%) patients underwent rethoracotomy for bleeding complications [9]. Furthermore, Doll et al. reported that 136/219 (63.5 \%) postcardiotomy patients who had undergone ECMO experienced severe mediastinal bleeding requiring rethoracotomy [10]. These data suggest that the underlying etiology and type of cannulation may influence the rate of bleeding complications.

Since post-cardiac arrest patients have better neurological outcomes when treated with induced hypothermia $[11,12]$, induced hypothermia therapy is often initiated together with ECPR. In the present study, all the patients underwent mild hypothermia therapy (target temp., $34{ }^{\circ} \mathrm{C}$ ). Hypothermia is associated with increased risk of bleeding $[13,14]$. Chest compression is also associated with bleeding 
Table 3 Comparison of cases with or without fatal bleeding

\begin{tabular}{llll}
\hline & $\begin{array}{l}\text { Fatal } \\
\text { bleeding }(-) \\
(n=29)\end{array}$ & $\begin{array}{l}\text { Fatal } \\
\text { bleeding }(+) \\
(n=3)\end{array}$ & $P$ value \\
\hline Age (mean) & 59.5 & 69.3 & 0.33 \\
Sex (male) & 25 & 2 & \\
Mean initial heparin dose (U/kg) & 54.6 & 44.7 & 0.42 \\
$\begin{array}{l}\text { Mean initial ACT (s) } \\
\text { Mean duration of conventional }\end{array}$ & 263.5 & 224.3 & 0.89 \\
CPR (min) & 51.9 & 67 & 0.18 \\
\hline
\end{tabular}

$A C T$ activated coagulation time, $C P R$ cardiopulmonary resuscitation

complications. The current recommendation for chest compression in patients in cardiac arrest is to push more than $3 \mathrm{~cm}$; however, chest compression exceeding $6 \mathrm{~cm}$ is associated with a greater incidence of complications [15]. Therefore, vigorous chest compression should be avoided in patients in whom ECPR will be introduced. Furthermore, compared with patients in acute respiratory failure receiving respiratory $\mathrm{ECMO}$, patients receiving ECPR, as well as postcardiotomy patients, have a higher risk of bleeding. An improved anticoagulation protocol is needed for these high-risk patients.

There were three cases of fatal bleeding related to chest compression among the patients examined in the present study. The mean initial heparin dose, mean initial ACT value, and mean duration of conventional CPR were not statistically associated with the incidence of fatal bleeding during ECMO (Table 3). Details of the factors contributing to these thoracic bleedings cannot be detected with this small-sized observational study; however, rib fractures can clearly be seen near the hematoma sites on computed tomography scans, so it is likely that chest compression was a contributing factor (Fig. 2). However, postmortem CT study for conventional CPR patients has shown that the incidence of rib fracture was $70 \%$, while hemothorax was seen in only $0.45 \%$ in the conventional CPR patients [16]. Since our patient group experienced 3 out of 32 (9.3\%) fatal chest hematoma, we assume that chest hematoma is exacerbated by our anticoagulation therapy for ECPR.

There are several reports indicating the safety of using a heparin-free ECMO circuit. Muehrcke et al. reported the details of 30 individual cases of heparin-free ECMO use; bleeding complications were reported in 12/30 (40\%) patients and 6 cases of intracardiac clotting were reported [17]. In a prospective analysis of 32 postcardiotomy patients receiving veno-arterial ECMO, Lamarche et al. reported that 14 (43.8\%) patients required rethoracotomy and that 2 patients experienced intracardiac thrombus [18]. Recently, there are several reports indicating IABP patients can be managed safely without heparin when used for postcardiotomy patients $[19,20]$. Together, these results suggest that the use of heparinfree circuits may lower the overall incidence of bleeding complications in postcardiotomy patients receiving ECMO.

Few studies have examined clotting events. However, Rastan et al. examined the findings of 78 autopsies of patients who had received ECMO. They reported thromboembolic findings in a total of $46.2 \%$ cases, $15.4 \%$ of which were known prior to death, indicating that clinically visible thromboembolic events occur much less frequently than subclinical thromboembolic events do [21]. In the present study, we did not examine thrombotic complications in detail; circuit clotting was only checked for by means of visual observation. It is, however, important to carefully monitor patients receiving ECMO for potential subclinical thromboembolic events.

The use of the anticoagulant nafamostat as an alternative to heparin is currently being investigated. Han et al. described the usefulness of nafamostat for both cardiac and respiratory ECMO; patients receiving nafamostat received less red blood cells and fresh frozen plasma compared with patients receiving heparin [22]. The incidence of haemorrhage or thrombosis was also lower in the nafamostat group. Further studies are needed to clarify how to avoid fatal bleeding in patients receiving ECPR.
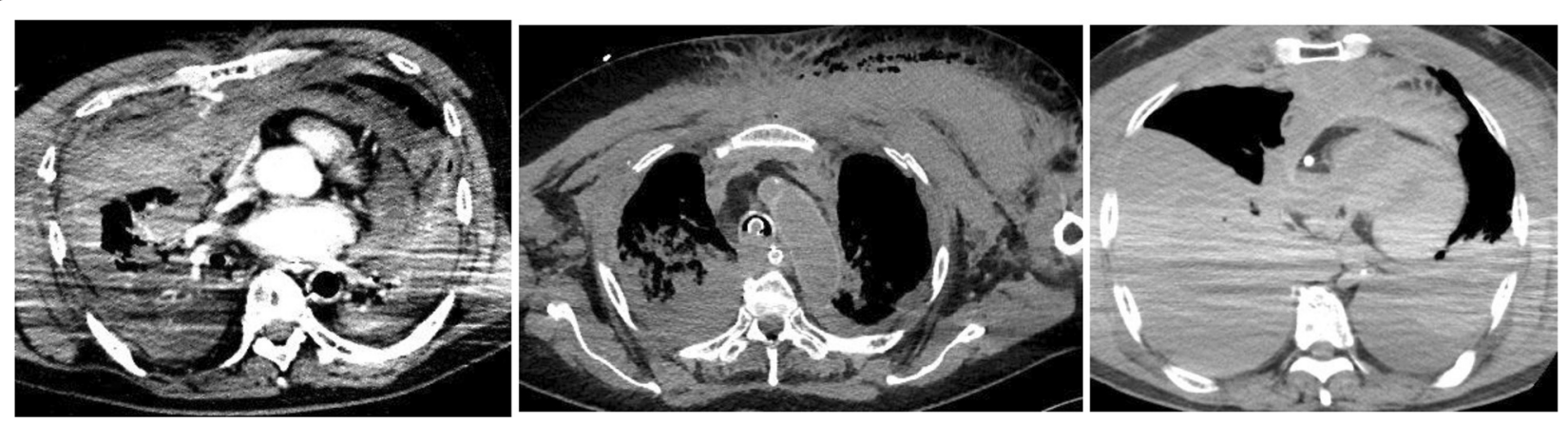

Fig 2 Computed tomography scans showing intra-thoracic, thoracic, and mediastinal bleeding, presumably caused by excessive chest compression, in the three patients in the present study who experienced fatal bleeding 


\section{Conclusions}

Fixed-dose heparin of 3000-U bolus resulted in a mean heparin dose per kilogram body weight of $53.6 \mathrm{U} / \mathrm{kg}$ and an ACT of $231.3 \mathrm{~s}$, and experienced 3 out of 32 fatal bleedings. Further research is warranted to optimize appropriate anticoagulation protocol for ECPR patients. At this point, avoiding vigorous chest compression and reducing anticoagulating agents are the possible intervention to minimize bleeding complications safely.

\section{Competing interests}

The authors declare that they have no competing interests.

\section{Authors' contributions}

YI drafted the manuscript. MY and MM contributed mechanical maintenance and setting of ECMO patients and also helped to make our anticoagulation protocol. AY and $\mathrm{KI}$ contributed to the patient treatment and making our anticoagulation protocol. HI drafted the manuscript and contributed the patient treatment. All authors read and approved the final manuscript.

\section{Acknowledgement}

The authors thank Drs. A. Masui, E. Kawamoto, T. Nakata, A. Shindo, K. Yokoyama, K. Suzuki, Y. Omori, T. Hatada, M. Fujioka, and T. Takeda, members of the Emergency and Critical Care Center, Mie University Hospital for their grateful contributions.

Received: 1 October 2014 Accepted: 1 July 2015

Published online: 21 July 2015

\section{References}

1. Sakamoto T, Morimura N, Nagao K, Asai Y, Yokota H, Nara S, et al. Extracorporeal cardiopulmonary resuscitation versus conventional cardiopulmonary resuscitation in adults with out-of-hospital cardiac arrest: a prospective observational study. Resuscitation. 2014;85:762-768.

2. Morimura N, Sakamoto T, Nagao K, Asai Y, Yokota H, Tahara Y, et al. Extracorporeal cardiopulmonary resuscitation for out-of-hospital cardiac arrest: a review of the Japanese literature. Resuscitation. 2011;82:10-4.

3. Lequier L, Annich GM, Massicotte P. Anticoagulation and bleeding during ECLS. In: Annich GM, Lynch WR, MacLaren G, Wilson JM, Bartlett RH, editors. Extracorporeal cardiopulmonary support in critical care. 4th ed. Michigan: Extracorporeal Life Support Organization; 2012. p. 157-70.

4. Field JM, Hazinski MF, Sayre MR, Chameides L, Schexnayder SM, Hemphill R, et al. Part 1: executive summary: 2010 American heart association guidelines for cardiopulmonary resuscitation and emergency cardiovascular care. Circulation. 2010;122:S640.

5. Hazinski MF, Nolan JP, Billi JE, Böttiger BW, Bossaert L, de Caen AR, et al. Part 1: Executive summary: 2010 International consensus on cardiopulmonary resuscitation and emergency cardiovascular care science with treatment recommendations. Circulation. 2010;122:S250.

6. Brogan TV, Thiagarajan RR, Rycus PT, Bartlett RH, Bratton SL. Extracorporeal membrane oxygenation in adults with severe respiratory failure: a multi-center database. Intens Care Med. 2009;35:2105-2114

7. Kushner FG, Hand M, Smith SC Jr, King SB 3rd, Anderson JL, Antman EM, et al. 2009 focused updates: ACC/AHA guidelines for the management of patients with ST-elevation myocardial infarction and ACC/AHA/SCAI guidelines on percutaneous coronary intervention. Circulation. 2009;120:2271-306.

8. Loh JP, Pendyala LK, Torguson R, Chen F, Satler LF, Pichard AA, et al. Incidence and correlates of major bleeding after percutaneous coronary intervention across different clinical presentations. Am Heart J. 2014;168:248-55.

9. Rastan AJ, Dege A, Mohr M, Doll N, Falk V, Walther T, et al. Early and late outcomes of 517 consecutive adult patients treated with extracorporeal membrane oxygenation for refractory postcardiotomy cardiogenic shock. J Thorac Cardiovasc Surg. 2010;139:302-11.

10. Doll N, Kiaii B, Borger M, Bucerius J, Krämer K, Schmitt DV, et al. Five year results of 219 consecutive patients treated with extracorporeal membrane oxygenation for refractory postoperative cardiogenic shock. Ann Thorac Surg. 2004;77:151-7.
11. Hypothermia after Cardiac Arrest Study Group. Mild therapeutic hypothermia to improve the neurologic outcome after cardiac arrest. $\mathrm{N}$ Engl J Med. 2002;346:549-56.

12. Bernard SA, Gray TW, Buist MD, Jones BM, Silvester W, Gutteridge G, et al. Treatment of comatose survivors of out-of-hospital cardiac arrest with induced hypothermia. N Engl J Med. 2002;346:557-63.

13. Michelson AD1, MacGregor H, Barnard MR, Kestin AS, Rohrer MJ, Valeri CR. Reversible inhibition of human platelet activation by hypothermia in vivo and in vitro. Thromb Haemost. 1994;71:633-40.

14. Reed RL 2nd, Bracey AW Jr, Hudson JD, Miller TA, Fischer RP. Hypothermia and blood coagulation: dissociation between enzyme activity and clotting factor levels. Circ Shock. 1990;32:141-52.

15. Hellevuo H1, Sainio M, Nevalainen R, Huhtala H, Olkkola KT, Tenhunen J, et al. Deeper chest compression-more complications for cardiac arrest patients? Resuscitation. 2013;84:760-5.

16. Kashiwagi Y, Sasakawa T, Tampo A, Kawata D, Nishiura T, Kokita N, et al. Computed tomography findings of complications resulting from cardiopulmonary resuscitation. Resuscitation. 2015;88:86-91.

17. Muehrcke DD, McCarthy PM, Stewart RW, Seshagiri S, Ogella DA, Foster RC, et al. Complications of extracorporeal life support systems using heparin-bound surfaces. J Thorac Car Diovasc Surg. 1995;110:843-51.

18. Lamarche $Y$, Chow B, Bédard A, Johal N, Kaan A, Humphries KH, et al. Thromboembolic events in patients on extracorporeal membrane oxygenation without anticoagulation. Innovations (Phila). 2010;5:424-9.

19. Kogan A, Preisman S, Sternik L, Orlov B, Spiegelstein D, Hod H, et al. Heparin-free management of intra-aortic balloon pump after cardiac surgery. J Card Surg. 2012;27:434-7.

20. Pucher PH, Cummings IG, Shipolini AR, McCormack DJ. Is heparin needed for patients with an intra-aortic balloon pump? Interact Cardiovasc Thorac Surg. 2012;15:136-40.

21. Rastan AJ, Lachmann N, Walther T, Doll N, Gradistanac T, Gommert JF, et al. Autopsy findings in patients on postcardiotomy extracorporeal membrane oxygenation (ECMO). Int J Artif Organs. 2006;29:1121-31.

22. Han SJ, Kim HS, Kim Kl, Whang SM, Hong KS, Lee WK, et al. Use of nafamostat mesilate as an anticoagulant during extracorporeal membrane oxygenation. J Korean Med Sci. 2011;26:945-50.

\section{Submit your next manuscript to BioMed Central and take full advantage of:}

- Convenient online submission

- Thorough peer review

- No space constraints or color figure charges

- Immediate publication on acceptance

- Inclusion in PubMed, CAS, Scopus and Google Scholar

- Research which is freely available for redistribution

Submit your manuscript at www.biomedcentral.com/submit
C) BioMed Central 